\title{
Fabrication of Division-of-focal-plane Polarizer Arrays by Electron Beam Lithography
}

\author{
Miao Yu, Li Li, Litong Dong, Lu Wang, Xing Chen, Zhengxun Song, Zhankun Weng, Zuobin Wang* \\ International Research Centre for Nano Handling and Manufacturing of China, \\ Changchun University of Science and Technology, Changchun 130022, China \\ wangz@cust.edu.cn
}

\begin{abstract}
The division-of-focal-plane (DoFP) polarizer is used to capture polarization information in real time without rotating the polarizer. In this paper, different periods of aluminum nano-wire grids with four different orientations offset by $45^{\circ}$ are fabricated using electron beam lithography (EBL) and inductively coupled plasma-reactive ion etching (ICP-RIE). The pitch of each unit is $7.4 \mu \mathrm{m}$ with a standard square. The transmission intensity measurement of DoFP arrays was presented to obtain the extinction ratios and transmission ratios which were used to evaluate the performance of grid polarizers.
\end{abstract}

Keywords-Division-of-focal-plane; nano-wire grid; EBL; polarizer

\section{INTRODUCTION}

Nature light contains the information of intensity, wavelength and polarization, the light reflected by object is polarized, so that the polarization information can help us to gain extra useful information, but human eyes are blind to it [1]. Polarizers can get the polarization information and get the polarization images by camera. Among the polarizers, metallic nano-wire polarizers are extensively used with the advantage of high extinction ratio [2]. When unpolarized light is incident onto the metal nano-wire gratings, the transverse electric (TE) wave is mostly reflected by the metal gratings, and the transverse-magnetic (TM) wave is mostly transmitted $[3,4,5]$. The ratio of transmitted TM and TE is the extinction ratio. Traditional polarization imaging works based on the division of time $[6,7]$ which changes the filter to capture images in different times, and the division of amplitude that the image is split into multiple paths. Each path has its own polarization optics and image plane [8,9]. The division of focal plane is a new method which is widely used in decades, and the schematic of DoFP polarizer arrays is shown in Fig. 1. DoFP sensors include imaging elements and nanowire polarization filters on the same substrate. The advantages of DoFP polarization sensors are compact and stabile, and it can capture all polarization information with an image in real time [10-16]. Numerous of approaches to the fabrication of DoFP metallic polarizer arrays have been proposed, such as laser interference lithography, nanoimprinting lithography $[17,18]$ and electron beam lithography [19,20]. However, laser interference lithography and nanoimprinting lithography are complex for four direction gratings. Electron beam lithography has a high resolution and a simple process, compared with other technologies.

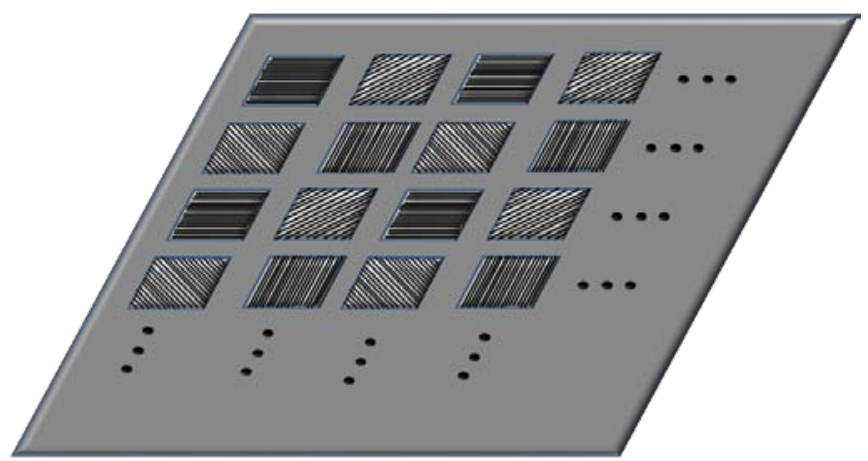

Fig. 1. Schematic of DoFP polarizer arrays.

In this paper, we focus on the fabrication of DoFP wiregrid polarizer arrays with the periods of $200 \mathrm{~nm}$ and $170 \mathrm{~nm}$ by EBL and ICP-RIE, which are used in polarization detection. Metal aluminum is used to fabricate the polarizers which has the best performance in the visible light [21]. The period of the grating should be smaller than the incident wavelength, so that the grating has only the zero-order diffraction wave [22]. The extinction ratios and transmission ratios of different periods were calculated in red wave band.

\section{METHOD AND FABRICATION}

\section{A. Fabrication Process}

The fabrication of DoFP polarizer arrays is carried out with EBL and ICP-RIE. The metal is aluminum and the periods are $200 \mathrm{~nm}$ and $170 \mathrm{~nm}$. The line widths are $100 \mathrm{~nm}$. The aluminum nano-wire grid with four different directions are $0^{\circ}, 45^{\circ}, 90^{\circ}$ and $135^{\circ}$. The pitch of each unit is $7.4 \mu \mathrm{m}$ with $1 \mu \mathrm{m}$ interval to reduce the cross talk between adjacent units. The fabrication process of DoFP polarizer arrays is shown in Fig. 2. and described as follows: 


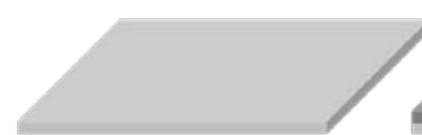

(a)

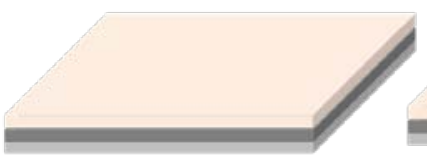

(c)

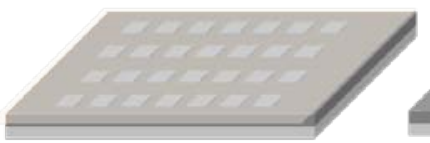

(e)

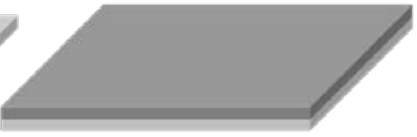

(b)

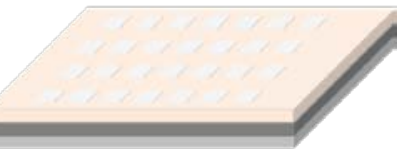

(d)

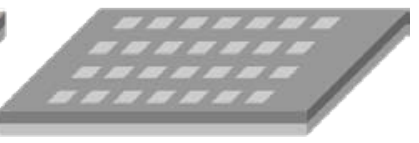

(f)
Fig. 2. Fabrication process of DoFP polarizer arrays. (a) Quartz glass substrate; (b) Aluminum is deposited on the substrate; (c) electric beam photoresist is spin coated on the surface of aluminum; (d) The designed patterns are exposed on the photoresist; (e) ICP-RIE; (f) The remaining photoresist is removed.

(1) A High transmittance quartz glass slide is used as the substrate of the DoFP polarizer. The quartz glass is cleaned by ultrasonic cleaning machine with deionized water, acetone and anhydrous alcohol, and dried by nitrogen.

(2) The glass substrate is coated with $150 \mathrm{~nm}$ aluminum layer, and the coating is compact and uniform.

(3) A $150 \mathrm{~nm}$ layer of positive electrobeam photoresist (AR-P 6200 ) is spin coated on the surface of aluminum at 4000rpm for $60 \mathrm{~s}$, and post baked at $180^{\circ} \mathrm{C}$ for $90 \mathrm{~s}$

(4) The DoFP patterns designed by CAD are exposed with electron beam lithography in the scanning electron microscope (FEI QUANTA FEG 250). The voltage is set as $30 \mathrm{kV}$, and the spot is set as 2. After the exposure, the sample is developed using developing liquid for 60 s, and washed using deionized water for 30s.

(5) Inductively coupled plasma-reactive ion etching (ICP-RIE) (SENTECH SI500) is used to transfer the developed patterns to the aluminum layer with $\mathrm{Cl}_{2}$ and $\mathrm{BCl}_{3}$ as the etching gasses to etch $\mathrm{Al}$ and $\mathrm{Al}_{2} \mathrm{O}_{3}$ for $80 \mathrm{~s}$.

(6) The remaining photoresist is removed by dispergator.

\section{B. Fabrication Result}

After the technological process listed in II.A, the fabricated DoFP polarizer arrays are observed by SEM, as shown in Fig. 3. Fig. 3(a) and Fig. 3(b) show the wire grids with four directions and the period is 200nm. Fig. 3(b) shows the details of Fig. 3(a). Fig. 3(c) shows the wire grids with the period of $170 \mathrm{~nm}$.

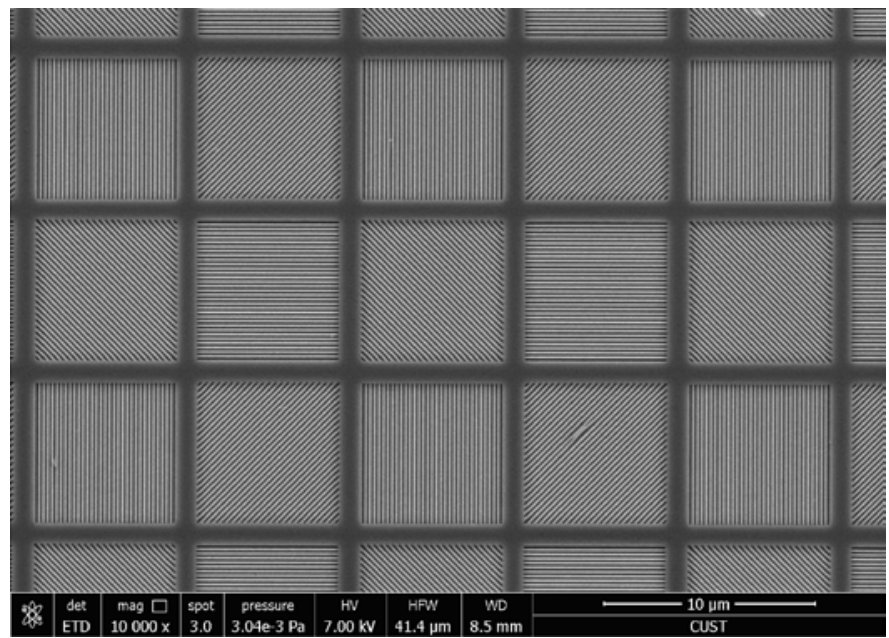

(a)

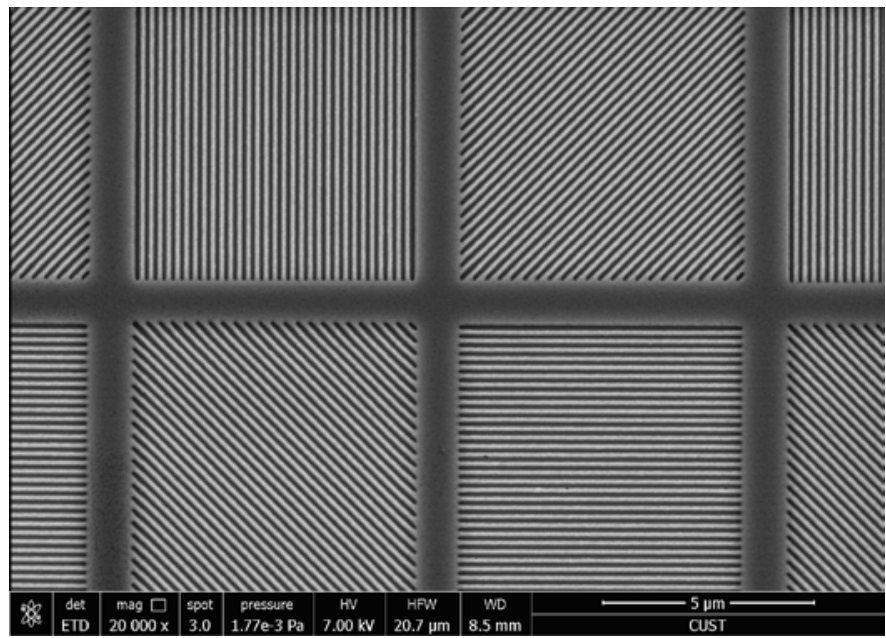

(b)

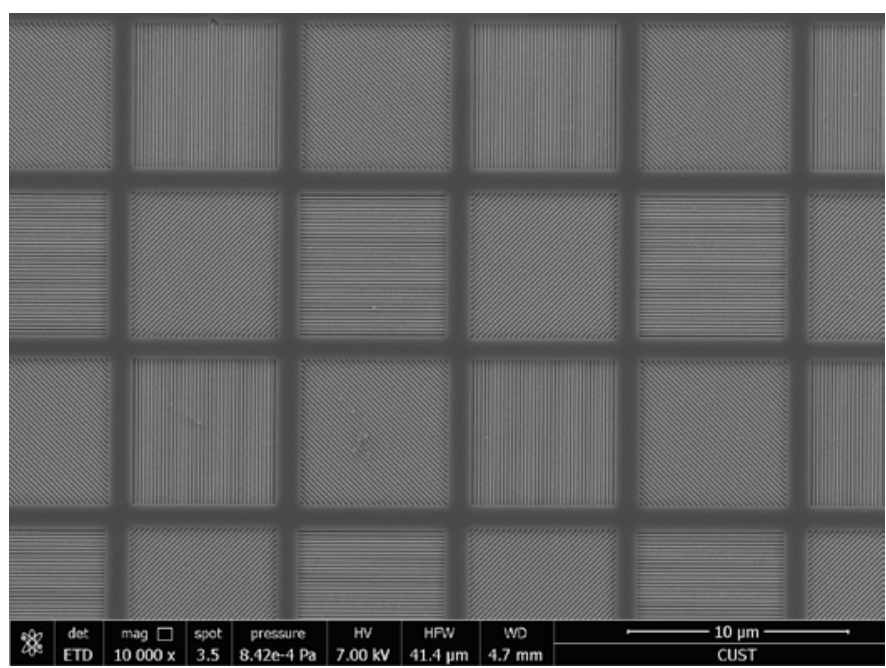

(c)

Fig. 3. Fabrication result of DoFP polarizer arrays observed by SEM. (a) and (b) The grids with the period of 200nm; (c) The grids with the period of $170 \mathrm{~nm}$. 
III. MEASUREMENTS AND DISCUSSIONS

A. Measurement Setup

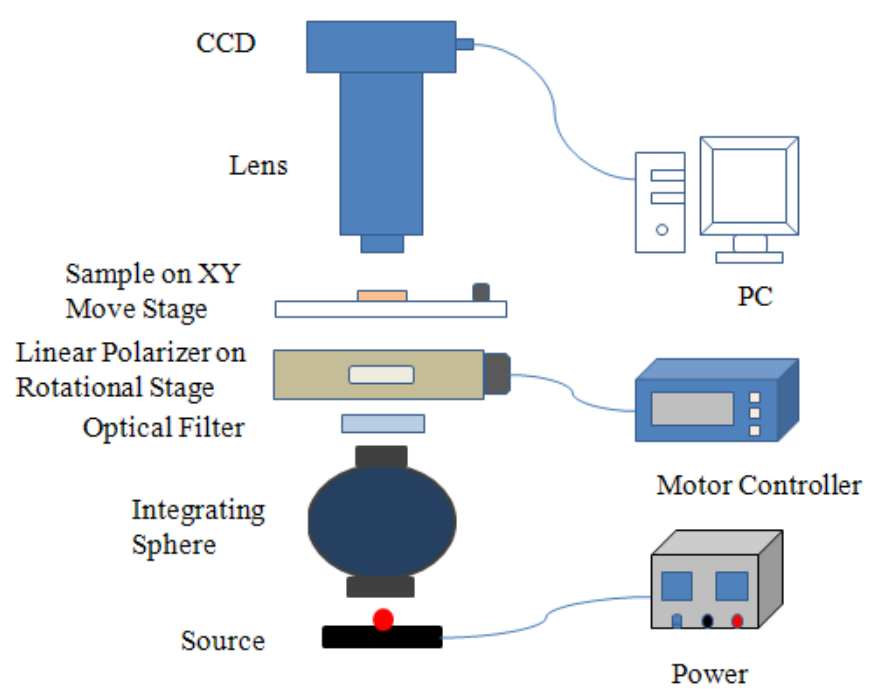

Fig. 4. Measurement setup for the measurement of DoFP polarizer arrays.

The measurement setup is shown in Fig. 4. The array of LEDs is used as the source to irradiate into a 4" integrating sphere to provide the uniform light intensity, and a narrow band filter (CWL=630, FWHM=10 ) is used to get a single wavelength source. The polarization angle of incident light is varied from $0^{\circ}$ to $180^{\circ}$ with an increment step of $5^{\circ}$ via an electric turntable controlled by a motor. The sample is put on the XY move stage and imaged with a CCD imaging sensor [23]. Fig. 5. show the CCD images of $200 \mathrm{~nm}$ period grids with the orientations at $0^{\circ}, 45^{\circ}, 90^{\circ}$ and $135^{\circ}$.

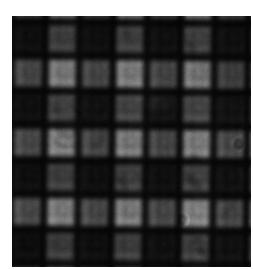

(a)

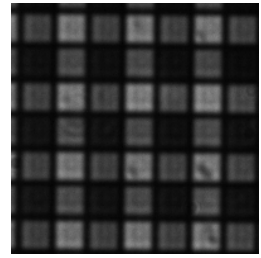

(c)

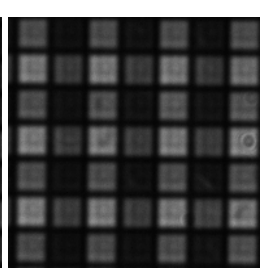

(b)

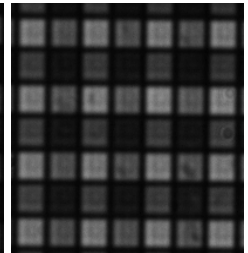

(d)
Fig. 5. The images are captured by CCD with four orientations (a) $0^{\circ}$ polarized light; (b) $45^{\circ}$ polarized light; (c) $90^{\circ}$ polarized light; (d) $135^{\circ}$ polarized light.

The DoFP polarizer array is measured to obtain the extinction ratio and the maximum and minimum transmission intensity responses. The extinction ratio is calculated by Eq. 1.[24] where $I_{\max }$ is the maximum transmission intensity response that the grid direction is parallel to the direction of fabricated arrays. $\mathrm{I}_{\min }$ is the minimum transmission intensity response that the grid direction is vertical to the direction of fabricated arrays.

\section{B. Measured Transmission Intensity}

The transmission intensity responses of four direction polarizer arrays are shown in Fig. 6. The transmission intensity distributions follow the Malus's law. The extinction ratio of $200 \mathrm{~nm}$ and $170 \mathrm{~nm}$ period gratings are 8.97 and 38.99. The maximum and the minimum polarization trasmittances of 200nm period are $65.63 \%, 7.31 \%$. The maximum and the minimum polarization trasmittances of $170 \mathrm{~nm}$ period are $76.75 \%, 1.97 \%$. The difference between different directions tansmission intensity responses are due to the fabrication result difference of each orientation.

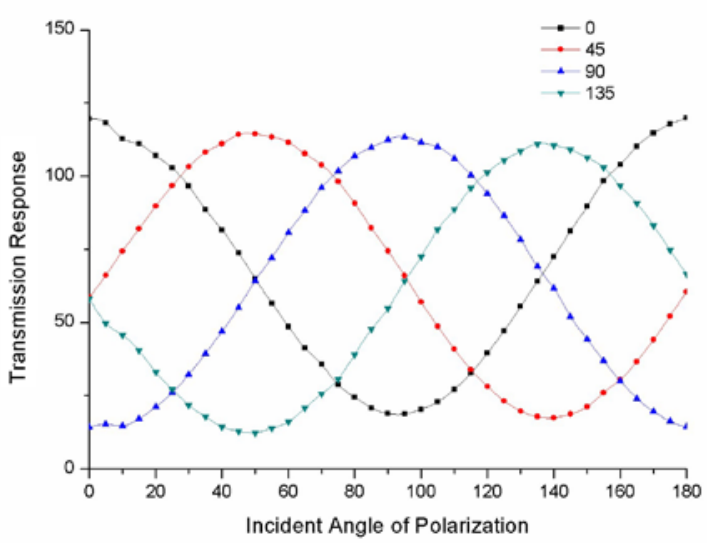

(a)

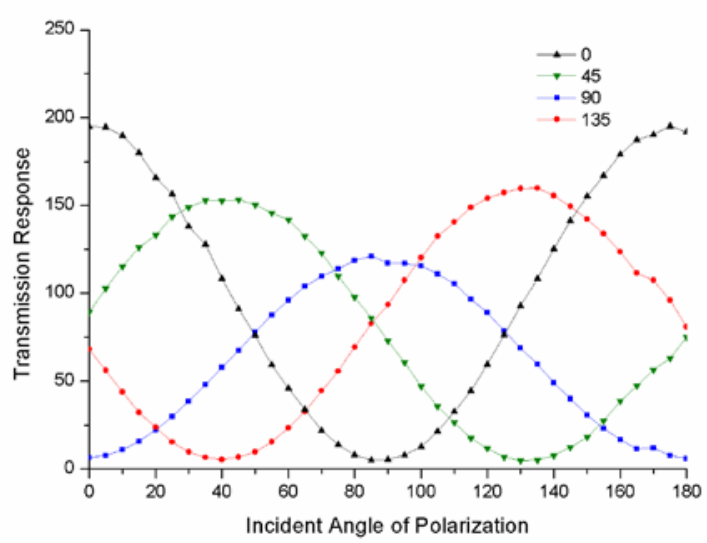

(b)

Fig. 6. Transmission intensity curves. (a) Period of 200nm; (b) Period of $170 \mathrm{~nm}$. 


\section{CONCLUSION}

In this paper, DoFP polarizer arrays with different periods are fabricated by EBL and ICP-RIE. The transmitted images of DoFP polarizer arrays are captured by a measurement setup, the responses of transmission intensities, extinction ratio and transmission ratio are obtained and calculated. The experiment results indicate that the period of nano-wire gratings will affect the performance of DoFP polarizers.

\section{ACKNOWLEDGMENT}

This work was supported by the "111" Project of China (D17017), National Key Basic Research Program of China (973 Program No.2012CB326406), EU FP7 (BioRA No.612641), China-EU H2020 (FabSurfWAR Nos.2016YFE0112100 and 644971), EU H2020 (MNR4SCell No.734174), National Natural Science Foundation Program of China (Nos.61176002, 11103047 and 11504030), and Jilin Provincial Science and Technology Program (Nos.20160520101JH, 20160101318JC and 20160623002TC).

\section{REFERENCES}

[1] Viktor Gruev, Rob Perkins and Timothy York, "Integrated High Resolution Division of Focal Plane Image Sensor with Aluminum Nanowire Polarization Filters,” Proc. of SPIE, vol. 7672, 76720G, 2010.

[2] Z.Y. Yang and Y.F. Lu, "Broadband nanowire-grid polarizers in ultraviolet-visible-near-infrared regions,” Optics express, vol. 15, pp. 9510-9519, 2007.

[3] Zhaoning Yu, Paru Deshpande, Wei Wu, Jian Wang, and Stephen Y. Chou, "Reflective polarizer based on a stacked double-layer subwavelength metal grating structure fabricated using nanoimprint lithography,” Applied physics letters, vol.7, pp. 927-929, 2000.

[4] Yasin Ekinci, Harun H. Solak, Christian David, and Hans Sigg, "Bilayer Al wire-grids as broadband and highperformance polarizers," Optics express, vol. 14, pp. 2323-2334, 2006.

[5] Thomas Weber, Thomas Käsebier, Ernst-Bernhard Kley and Andreas Tünnermann, "Broadband iridium wire grid polarizer for UV applications,” Optics letters, vol. 36, pp. 445-447, 2011.

[6] D. H. Goldstein, "Mueller matrix dual-rotating retarder polarimeter," Applied optics, vol. 31, pp. 6676-6683, 1992.

[7] K. J. Voss, and Y. Liu, "Polarized radiance distribution measurements of skylight. I. System description and characterization,” Applied Optics, vol. 36, pp. 6083-6094, 1997.

[8] C. J. Zappa, M. L. Banner, H. Schultz, A. Corrada-Emmanuel, L. B. Wolff, and J. Yalcin, "Retrieval of short ocean wave slope using polarimetric imaging,” Meas. Sci. Technol. Vol. 19, 055503, 2008.

[9] S. Bear Powell and Viktor Gruev, "Calibration methods for division-offocal-plane polarimeters ,” Optics express, vol. 21, pp. 21039-21055, 2013.
[10] Viktor Gruev and Rob Perkins, "A 1 MPixel CCD Image Sensor with Aluminum Nanowire Polarization Filter,” Proc. of IEEE, pp. 629-632, 2010.

[11] Viktor Gruev, Jan Van der Spiegel and Nader Engheta, "Dual-tier thin film polymer polarization imaging sensor,” Optics express, vol. 18, pp. 19292-19303, 2010.

[12] Robert Perkins and Viktor Gruev, "Signal-to-noise analysis of Stokes parameters in division of focal plane polarimeters," Optics express, vol. 18, pp. 25815-25824, 2010.

[13] Shengkui Gao and Viktor Gruev, "Bilinear and bicubic interpolation methods for division of focal plane polarimeters," Optics express, vol. 19, pp. 26161-26173, 2011.

[14] Shengkui Gao and Viktor Gruev, "Gradient-based interpolation method for division-of-focal-plane polarimeters,” Optics express, vol. 21, pp. 1137-1151, 2013.

[15] Zhigang Zhang, Fengliang Dong, Teng Cheng, Kang Qiu, Qingchuan Zhang, Weiguo Chu, and Xiaoping Wu, "Nano-fabricated pixelated micropolarizer array for visible imaging polarimetry," Review of scientific instruments, vol. 85, 105002, 2014.

[16] Timothy York,1 Radoslav Marinov,2 and Viktor Gruev, "260 framesper-second $648 \times 488$ resolution division-of-focal-plane polarimeter with structural dynamics and tracking applications,” Optics express, vol. 24, pp. 8243-8252, 2016.

[17] Seh-Won Ahn, Ki-Dong Lee, Jin-Sung Kim, Sang Hoon Kim, Joo-Do Park, Sarng-Hoon Lee and Phil-Won Yoon, "Fabrication of a $50 \mathrm{~nm}$ half-pitch wire grid polarizer using nanoimprint lithography," Nanotechnology, vol. 16, pp. 1874-1877, 2005.

[18] Se Hyun Ahn, Jin-Sung Kim, and L. Jay Guo, "Bilayer metal wire-grid polarizer fabricated by roll-to-roll nanoimprint lithography on flexible plastic substrate,” J. Vac. Sci. Technol. B, vol. 25, pp. 2388-2391, 2007.

[19] Jian Jim Wang, Frank Walters, Xiaoming Liu, Paul Sciortino, and Xuegong Deng, "High-performance, large area, deep ultraviolet to infrared polarizers based on $40 \mathrm{~nm}$ line/78 nm space nanowire grids," Applied physics letters, vol. 90, 061104, 2007.

[20] ZHANG Zhi-Gang, DONG Feng-Liang, CHENG Teng, QIAN Ke-Mao, QIU Kang, ZHANG Qing-Chuan, CHU Wei-Guo, WU Xiao-Ping, "Electron Beam Lithographic Pixelated Micropolarizer Array for RealTime Phase,” CHIN. PHYS. LETT., vol. 31, 114208, 2014.

[21] Z. Y. Yang, M. Zhao, N. L. Dai, G. Yang, H. Long, Y. H. Li, and P. X. $\mathrm{Lu}$, "Broadband Polarizers Using Dual-Layer Metallic Nanowire Grids," IEEE photonics technology letters, vol. 20, pp. 697-699, 2008.

[22] Fantao Meng, Jinkui Chu, Zhitao Han and Kaichun Zhao, "The Design of the Sub-Wavelength Wire-Grid Polarizer," Proceedings of the 7th IEEE International Conference on Nanotechnology, pp. 942-946, 2007.

[23] Shengkui Gao, Raphael Njuguna, and Viktor Gruev, "Fabrication and performance evaluation of pixelated nano-wire grid polarizer, ” Proc. of SPIE, vol. 8873, 88730L, 2013.

[24] Sanshiro Shishido, Toshihiko Noda, Kiyotaka Sasagawa, Takashi Tokuda, and Jun Ohta, "Polarization Analyzing Image Sensor with OnChip Metal Wire Grid Polarizer in 65-nm Standard Complementary Metal Oxide Semiconductor Process,” Japanese journal of applied physics, vol. 50, 04DL01, 2011. 